\title{
Ionospheric corrections via PIM and real-time data
}

\author{
R.M. Campbell \\ Netherlands Foundation for Research in Astronomy, Postbus 2, \\ 7990 AA dwingeloo, The Netherlands
}

\begin{abstract}
We describe a method for removing ionospheric effects from single-frequency radio data a posteriori. This method is based on a theoretical climatological model developed by the USAF, which returns $n_{e}(\vec{r}, t)$ along the line of sight to the source. Together with a model of $\mathbf{B}_{\oplus}$, ionospheric delay and Faraday rotation values ensue. If contemporaneous ionospheric data - GPS TEC observations or ionosonde profiles - exist, they can be incorporated to update the modeled $n_{e}$.
\end{abstract}

Key words: techniques: interferometric, plasma, atmospheric effects PACS: 94.20.Bb, 94.20.Dd, 95.75.-z, 95.75.Kk

\section{Introduction}

The Earth's ionosphere imparts additional phase/group delay $\left(\tau_{\text {iono }}\right)$, Faraday rotation $\left(\psi_{\text {iono }}\right)$, refraction, and absorption to cosmic radio signals propagating through it. These ionospheric effects arise because of the dispersive index of refraction for a cold, weak plasma (and the presence of Earth's magnetic field in the case of Faraday rotation). For $\nu \geq 10 \mathrm{MHz}$, we can safely ignore absorption (see, e.g., Davies (1990), §7.4.1), and will do so henceforth. We can then write the collision-free Appleton-Hartree index of refraction:

$$
\mu_{p}^{2}=\frac{2 X(1-X)}{2(1-X)-Y_{\perp}^{2} \pm\left[Y_{\perp}^{4}+4(1-X)^{2} Y_{\|}^{2}\right]^{\frac{1}{2}}}
$$

where $X \equiv\left(e^{2} / 4 \pi^{2} \varepsilon_{0} m_{e}\right) n_{e} / \nu^{2}, Y \equiv\left(e / 2 \pi m_{e}\right) B / \nu$, and the " $\perp$ " and " $\| "$ subscripts for $Y$ refer to $B_{\perp}$ and $B_{\|}$, the components of the Earth's magnetic field perpendicular and parallel to the direction of propagation. The " \pm " shows that there are two characteristic modes, giving rise to Faraday rotation for linear polarizations. Equation (1) can be expanded to yield $\mu_{p}$ to the desired 
order (except for $\nu$ well less than $50 \mathrm{MHz}$ at times of solar maximum, firstorder expansion will suffice). Any ionospheric effect will then be proportional to $\int f\left(n_{e}, B_{\|}, B_{\perp}\right) d l$, where the integration is along the propagation path. To first order, $\tau_{\text {iono }} \propto \int n_{e} d l[\equiv \mathrm{TEC}]$, and $\psi_{\text {iono }} \propto \int n_{e} B_{\|} d l$.

Because ionospheric effects are dispersive, dual-frequency observations are the most straightforward way of correcting for them (again, to first order). However, such a tactic is not conducive to some types of observations (e.g., should a source's spectral index preclude X-band detection). This paper will discuss one approach for removing ionospheric contributions from single-frequency data. This approach comprises two components: an ionospheric "climatology" model that derives $n_{e}(\vec{r}, t)$ and the capability to incorporate contemporaneous ionospheric data, if available, to update the modeled $n_{e}$.

\section{PIM: Parameterized Ionospheric Model}

PIM is a theoretical model of ionospheric climatology developed at USAF Phillips Laboratory. It forms the basis for PRISM, a global real-time ionospheric model for operational use at the Air Force Space Forecast Center (see $\S 3)$. PIM compiles runs of the more general Phillips Lab Global Theoretical Ionospheric Model (GTIM), spanning parameter space in local time, latitude, season, solar activity, geomagnetic activity, and interplanetary magnetic-field direction. GTIM calculates $n_{e}$ by solving ion-balance equations along magnetic flux tubes, taking into account production, loss, and transport processes. Daniell et al. (1995) describes PIM itself; Anderson (1993a) and Anderson et al. (1996) discuss the physics underlying GTIM. These references also discuss the operational (and philosophical) differences between theoretical and empirical climatologies.

To illustrate representative ionospheric morphology in PIM, Fig. 1 shows a global map of vertical TEC at 20UT around the vernal equinox and solar maximum. The contours are labeled in units of TECU $\left(=10^{16} \mathrm{~m}^{-2}-\right.$ a rule of thumb: $1 \mathrm{TECU} \simeq \frac{4}{3 \nu}$ cycles of ionospheric delay, for $\nu$ in $\mathrm{GHz}$ ). The thick vertical line represents the longitude of the sun, and the thick dashed line is the magnetic equator. Roughly speaking, the sun "pulls" the entire pattern, sliding along the magnetic equator, from east to west. We can see that the predicted horizontal gradients have significant structure in both $\mathrm{E}-\mathrm{W}$ and $\mathrm{N}-$ $\mathrm{S}$ directions, especially for lines of sight transecting low-latitude regions. The blobs of anomalously high TEC lying at $\Phi \sim \pm 15^{\circ}$ during the local evening illustrate the interplay between production, loss, and transport processes in the ionosphere. Neutral winds in the early afternoon in equatorial regions tend westward. The differential collisional coupling of the neutrals to electrons and ions generates an eastward horizontal $\mathbf{E}$. Acting with the northward $\mathbf{B}_{\oplus}$, also predominantly horizontal near the equator, an $\mathbf{E} \times \mathbf{B}$ drift pushes electrons up, 
to regions of lower neutral densities, and hence slower recombination (loss). These blobs of electrons fall back down along lines of $\mathbf{B}_{\oplus}$, moving away from the magnetic equator and persisting a few hours longer than they would have otherwise. This process also causes a diurnal variation in the height of maximum electron density $\left(h_{\mathrm{m}} F_{2}\right)$ typically in a range of $250-400 \mathrm{~km}$. A general weakness of vertical TEC maps like Fig. 1 is that they mask the fact that vertical $n_{e}$ profiles can change significantly, in their shape and $h_{\mathrm{m}} F_{2}$, on time-scales corresponding to diurnal, seasonal, and solar-cycle factors.

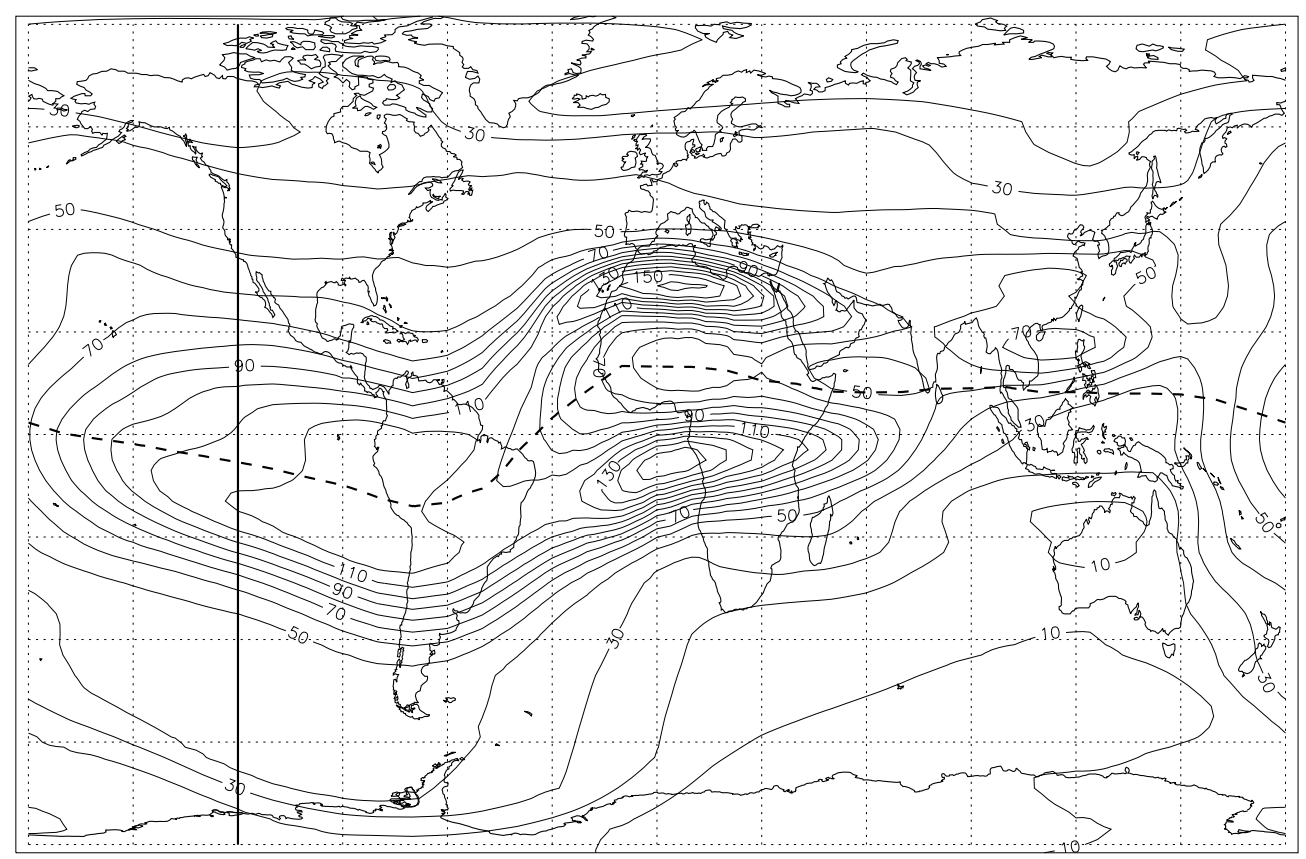

Fig. 1. Vertical TEC calculated by PIM at 20UT for the vernal equinox during solar maximum, in units of TECU. The longitude grid marks correspond to 2-hr segments of local mean time; the latitude grid marks fall every $20^{\circ}$. The thick dashed line represents the magnetic equator; the thick vertical line the longitude of the sun.

I am working on a variant of PIM, originally motivated by application to VLBI astrometry (unimaginatively, called PIMVLBI). The user needs only to provide the coordinates of the radio antennas and sources, and the JD/UT of observations (e.g., input of a MkIII/HOPS export file will suffice to pass all necessary information). The program automatically looks up the relevant geophysical/solar parameters from files downloaded from NOAA and GSFC. Internally, PIM computes $n_{e}$ at sample points along the propagation path from the source; PIMVLBI includes IGRF routines to calculate $B_{\|}$and $B_{\perp}$ at these points. The slant $\tau_{\text {iono }}$ and $\psi_{\text {iono }}$ can be integrated from these values. This approach takes directly into account the more realistic horizontal gradients and vertical profiles in PIM, obviating the need for purely geometric slant factors about fixed-height sub-ionospheric points, as are "traditionally" used for converting vertical to slant TEC. 


\section{Real-Time Data}

There are, however, processes whose effects PIM does not model — global magnetic storms, traveling ionospheric disturbances, scintillation (see, e.g., Davies (1990)). For this reason, inclusion of contemporaneous ionospheric observations should improve the reliability of the modeled $n_{e}$. Much of the data used to constrain PRISM (Anderson, 1993b) are available only to the military (as is the PRISM code itself). I have rather incorporated a real-time adjustment (RTA) algorithm devised for range/refraction corrections at an ARPA radar in the Marshall Islands (Daniell et al., 1996). This RTA distinguishes between two types of real-time data: integral and profile.

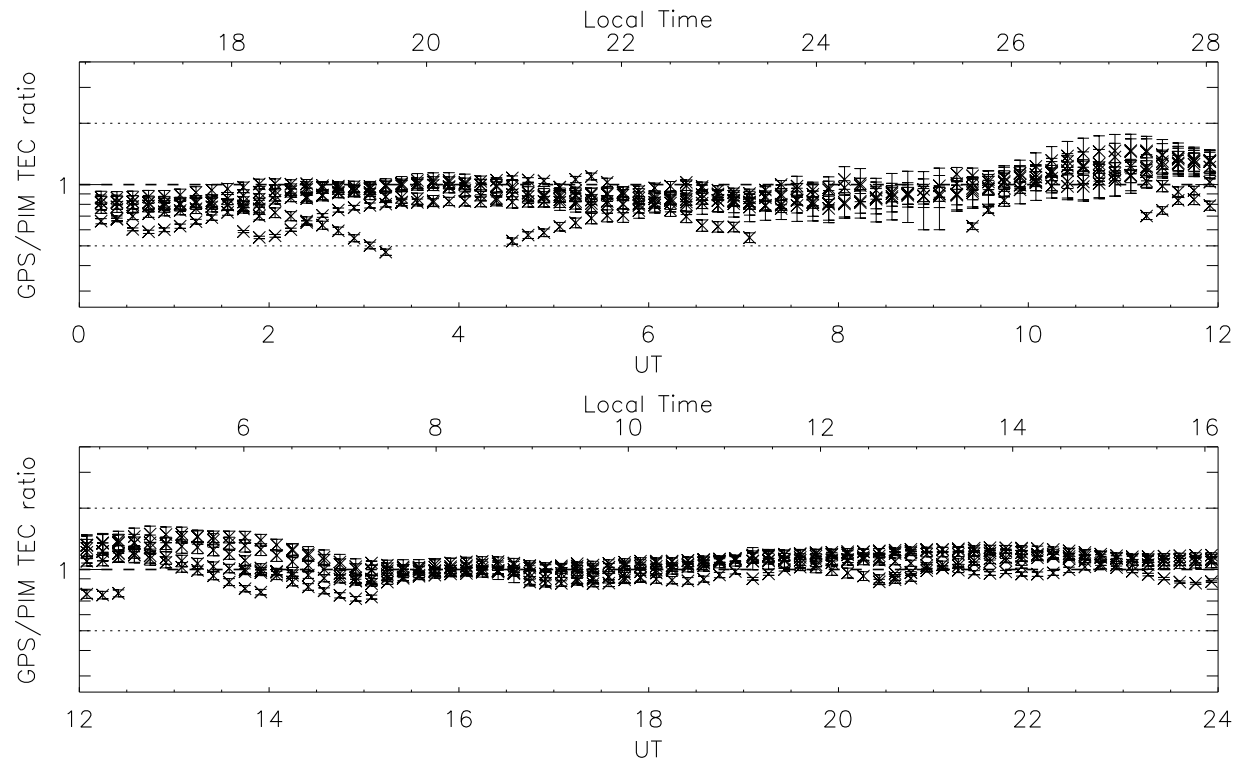

Fig. 2. Ratio of GPS-observed to PIM-predicted TEC from Goldstone on 9 June 1998. The $y$-axis is logarithmic, with dotted lines at ratios of 0.5 and 2 . The local time at Goldstone is labeled on the upper axes.

TEC observations from a collocated GPS receiver would be an example of integral data, providing a ratio of GPS/PIM TEC along the line of sight to each observed GPS satellite (SV). In this situation, one may view PIM as a physics-based mapping function for spatial interpolation among the GPS TEC values to the direction of the source. Fig. 2 plots the GPS/PIM TEC ratios observed from the IGS receiver at Goldstone on 9 June 1998. For legibility, the 30s-sampled IGS data has been culled to 10-minute sampling. The error bars stem from the uncertainty in the GPS TEC measurements, for which I use the minimum of the running-30-minute standard deviation in the fit of differential carrier phase and code range for each satellite pass. Ratios for all observed SVs are plotted regardless of their direction. If PIM has reproduced all spatial gradients perfectly, each such ratio would equal a single constant. Scatter within the ratios at a specific time therefore implies unmodeled structure in $(A z, E l)$ space. We can see that there are a couple individual SVs diverging 
from the trend between 18-22 LT on that day; these lie at low elevations off to the south-west, sampling the equatorial anomaly. Except for these cases, the GPS/PIM ratio behaves well, especially so from 08-16 LT.

Ionosondes would be a representative source of profile data. The RTA currently uses $h_{\mathrm{m}} F_{2}$ and the maximum density $\left(N_{\mathrm{m}} F_{2}\right)$ from the ionosonde profile. Data from ionosondes can neatly complement GPS TEC constraints. One problem with the latter is that SVs may not lie along the lines of sight you would prefer to sample at a given time. In principle, an ionosonde station could be located where ionospheric effects along specific lines of sight from a telescope would be most sensitive to changes in $h_{\mathrm{m}} F_{2}$ and $N_{\mathrm{m}} F_{2}$. For example, $h_{\mathrm{m}} F_{2}$ rises towards $400 \mathrm{~km}$ in the equatorial anomaly. Low elevation southerly lines of sight from Europe (as far north as WSRT) intersect this altitude around the African Mediterranean coast — just where Fig. 1 shows the equatorial anomaly in the evening. There are UMass/Lowell digisondes (digital ionosondes) in southern Spain and Italy (Klobuchar, 1997); access to their data could increase the reliability of PIMVLBI $n_{e}$ estimates along such directions for EVN stations, regardless of whether an SV happened to be conveniently located. Other types of profile-data instruments would include incoherent scatter radars and occultations of low-earth orbiting satellites carrying a GPS receiver.

There are also other possibilities for expanding the Daniell et al. (1996) RTA. This was developed to provide real-time corrections for a site literally in the middle of the ocean; the typical situation for a radio-astronomy antenna providing data for later VLBI correlation is not so severe on both counts. PIMVLBI already takes advantage of the relaxation of the real-time requirement by using data from the complete passes of relevant SVs when computing GPS TEC values at a specific time (allowing us to use low-elevation GPS satellites with noise characteristics comparable to higher-elevation ones when anti-spoofing is on; conversely, Daniell et al. (1996) used no GPS satellite with $E l<35^{\circ}$ ). However, the PIMVLBI RTA still considers the GPS/PIM ratio "field" as an independent spatial function each instant. This leads to situations where, say, an SV yields a non-unity GPS/PIM ratio at a time just before it sets. Immediately afterwards, the information contained in the ratio is lost, even though the actual ionospheric $n_{e}$ distribution is not changing so abruptly. An improved algorithm might be able to incorporate some sort of physically reasonable temporal smoothing of the spatial ratio-field in response to such discontinuous changes in the SV sampling. Further, we may also be able to incorporate GPS data from non-collocated receivers nearby. GPS satellites unfortunately move too slowly to allow proper tomographic reconstruction of $n_{e}$ above an array of receivers (as could be done with the old TRANSIT satellites for meridional arrays), but inclusion of PIM and ionosonde $h_{\mathrm{m}} F_{2}$ and $N_{\mathrm{m}} F_{2}$ data could perhaps provide sufficient external constraints to increase the reliability of the slant-to-vertical and horizontal-translation transformations required to use non-collocated GPS/PIM ratios in the traditional way. 


\section{Concluding Thoughts}

The aeronomy community has made a great deal of progress in ionospheric modeling since the mid-70's that has not been thoroughly absorbed by radio astronomy, and from which we could profit. The PIM model just described falls in this category. Among its advantages, it is freely available and is upgraded as model refinements proceed. It calculates $n_{e}(\vec{r})$, allowing direct integration, to arbitrary order, of effects along the specific lines of sight from our antennas to our sources. It can readily incorporate various types of external contemporaneous data should they be available, but does not require them should they not. The next step, once all the code is in place, involves pursuing validation, especially via the extensive store of IGS GPS data (e.g., more "Figures 2") to characterize its strengths and weaknesses as correlated to location, local time, season, solar cycle, etc. Such work would complement USAF PRISM-validation efforts, for which GPS data play a decidedly subsidiary role to ionosondes and in situ ionospheric data. There has also been preliminary discussion about including ionospheric modeling capability in AIPS++. For example (in broadest strokes of AIPS++ parlance), the PIM databases and geophysical-parameter files could become global data; GPS TEC measurements from individual stations could become part of their measurement set; and the user could have the flexibility to explore various combinations of PIM and the available real-time data as she sees fit during the course of analysis.

\section{References}

Anderson, D.N., 1993a, in: Matsumoto, H. (ed), Modern Radio Science 1993, Oxford University Press, Oxford, p. 159.

Anderson, D.N., 1993b, in: Goodman, J.M. (ed), Proceedings of the 1993 Ionospheric Effects Symposium, NRL, Washington, DC, p. 353.

Anderson, D.N., Decker, D.T., \& Valladares, C.E., 1996, in: Schunk, R.W. (ed), Solar-Terrestrial Energy Program: Handbook of Ionospheric Models, NOAA, Boulder, p. 133.

Campbell, R.M., 1995, Ph.D. Thesis, Harvard University.

Daniell, R.E., Brown, L.D., Anderson, D.N., Fox, M.W., Doherty, P.H., Decker, D.T., Sojka, J.J., \& Schunk, R.W., 1995, Radio Sci., 30, 1499.

Daniell, R.E., Millman, G.H., Hunt, S.M., Brown, L.D., Lamicela, J.T., \& Sponseller, D.L., 1996, in: Goodman, J.M. (ed), Proceedings of the 1996 Ionospheric Effects Symposium, NRL, Washington, DC, p. 368.

Davies, K., 1990, Ionospheric Radio, Peter Peregrinus, Ltd., London.

Klobuchar, J.A., 1997, Radio Sci., 32, 1943. 